\title{
P-process nucleosynthesis in detonating white dwarfs in the light of multidimensional hydrodynamical models
}

\author{
S. Goriely ${ }^{1}$, D. García-Senz ${ }^{2,3}$, E. Bravo ${ }^{2,3}$, and J. Joséé,3
}

\author{
${ }^{1}$ Institut d'Astronomie et d'Astrophysique, Université Libre de Bruxelles, CP 226, 1050 Brussels, Belgium \\ e-mail: sgoriely@astro.ulb.ac.be \\ 2 Departament de Física i Enginyeria Nuclear, UPC, Sor Eulalia d'Anzizu s/n, B5, 08034 Barcelona, Spain \\ e-mail: [domingo.garcia; jordi .jose; eduardo.bravo] @upc. edu \\ 3 Institut d'Estudis Espacials de Catalunya, Ed. Nexus-201, C/ Gran Capitá 2-4, 08034 Barcelona, Spain
}

Received 30 June 2005 / Accepted 11 September 2005

\section{ABSTRACT}

The p-process nucleosynthesis in He-accreting white dwarfs with sub-Chandrasekhar mass is revisited in the light of multi-dimensional hydrodynamical simulations. Post-processing calculations are performed on a sample of well-chosen track particles representative of the core and envelope material that is subject to the p-process. The p-abundance distributions in the disrupted core as well as in the ejected envelope are estimated and compared with previous analysis based on spherical-symmetric simulations. The present calculations confirm the results obtained in 1D simulations and the possibility to produce in particular the puzzling Mo and Ru p-isotopes in the envelope, provided it is initially enriched in s-process elements.

Key words. hydrodynamics - nuclear reactions - nucleosynthesis - stars: white dwarfs

\section{Introduction}

The explosion of a moderately massive white dwarf (WD) as a result of a sequence of detonations resulting from the accretion of He-rich matter has recently deserved some attention as a possible site for the p-process nucleosynthesis (Goriely et al. 2002). For some time that scenario was also considered as a plausible alternative to the standard model for type Ia Supernovae owing to the more credible evolutionary track leading to the ignition of the detonation and their relatively high estimated frequency of about $0.01 \mathrm{y}^{-1}$ (Iben \& Tutukov 1991). Nevertheless the resulting spectrum of the explosions near maximum light does not agree so well with observations as it does for the explosion of a WD approaching the Chandrasekhar-mass limit. Recent simulations (Yoon \& Langer 2004) also showed that the He-detonation may not take place when including the effects of rotational spin-up of the accreting WD, at least for high mass accretion rates of the order of $10^{-8} M_{\odot} \mathrm{y}^{-1}$. For these reasons, there is now a wide agreement that the sub-Chandrasekhar type-I supernova class does not constitute the dominant scenario for these explosions although it could explain a small fraction of them. From the nucleosynthetic point of view, however, the explosion of subChandrasekhar-mass WD have interesting features. These arise in part because the initial off-center ignition propagates as a detonation through the He-rich envelope leading to the synthesis of a noticeable amount of $\alpha$-elements. Hydrodynamical calculations show that besides the more abundant species, ${ }^{56} \mathrm{Ni}$ and unburnt $\mathrm{He}$, an overproduction of ${ }^{44} \mathrm{Ca},{ }^{47,48} \mathrm{Ti}$, and ${ }^{51} \mathrm{~V}$ is found (Woosley \& Weaver 1994) which are deficient in current Galactic chemical evolution models. It has been suggested that off-center helium detonations could represent the major source to the synthesis of the radioactive isotope ${ }^{44} \mathrm{Ti}$ in the Galaxy (Timmes et al. 1996), making this scenario relevant to $\gamma$-ray astronomy. In addition, the sub-Chandrasekharmass explosion scenario could also be an important site for the p-process nucleosynthesis and in particular of the puzzling light $\mathrm{p}$-isotopes of $\mathrm{Mo}$ and $\mathrm{Ru}$, as shown by Goriely et al. (2002, 2003).

So far, all the aforementioned hydrodynamical calculations were carried out assuming spherical symmetry. This is, however, not very realistic since the ignition is expected to start in one or several discrete regions rather than in a spherical shell. The main purpose of this letter is to explore the robustness of the results presented by Goriely et al. (2002) concerning the synthesis of p-elements when the hypothesis of spherical symmetry is removed and the evolutionary tracks obtained from a full hydrodynamical calculation in 3D are considered. The 3D simulation is carried out using the smoothedparticle-hydrodynamics (SPH) technique in conjunction with a reduced nuclear network. The main features of the multidimensional hydrodynamical method, the physics and initial model considered as well as a brief summary of the hydrodynamical 
Table 1. Characteristics of the initial model (masses in $M_{\odot}$ ). The parameters are: central density and temperature (in $10^{6} \mathrm{~g} \mathrm{~cm}^{-3}, 10^{7} \mathrm{~K}$, respectively), core radius (in $10^{8} \mathrm{~cm}$ ), values of $\rho$ and $T$ at the core boundary, core mass and chemical composition, helium layer mass and composition, total radius and initial binding energy (in $10^{50} \mathrm{ergs}$ ).

\begin{tabular}{rrrrrrrrrrrrr}
\hline \hline$\rho_{c 6}$ & $T_{7 c}$ & $R_{c 8}$ & $\rho_{b 6}$ & $T_{b 7}$ & $M_{\mathrm{c}}$ & $X_{\mathrm{C}}$ & $X_{\mathrm{O}}$ & $X_{\mathrm{Ne}}$ & $M_{\mathrm{He}}$ & $X_{\mathrm{He}}$ & $R_{\star 8}$ & $\mathrm{BE}_{50}$ \\
\hline 37.4 & 0.45 & 3.35 & 4 & 10 & 0.8 & 0.3 & 0.69 & 0.01 & 0.22 & 1 & 5.3 & -1.803 \\
\hline
\end{tabular}

evolution of the model are given in Sect. 2. In Sect. 3, a postprocessing nucleosynthesis calculation with a large network is used to set the detailed composition of the ejected material. Conclusions are drawn in Sect. 4.

\section{Hydrodynamical evolution of the exploding WD}

There are few multidimensional calculations concerning the detonation of a WD with a mass well below the Chandrasekharmass limit. The most recent and complete are the 2D simulations described in Livne \& Arnett (1995) and several 3D calculations presented in García-Senz et al. (1999) (hereafter, GSBW). In these models the detonation starts either in a pointlike region of the star or in several discrete points chosen at random, always on the interphase between the carbon-oxygen and helium layers. The main conclusion of both papers is that despite the very different hydrodynamical paths followed by these multidimensional models the gross features of the isotopic yields are rather similar. Nevertheless it is unclear if these conclusions can be extended to all nuclides, especially to those synthesized by the p-process.

The hydrodynamical evolution of the exploding WD is followed without any geometrical constraint using a SPH code especially suited to follow a detonation in degenerate material. Such hydrocode incorporates ellipsoidal interpolating kernels to improve the description of the shock front, an accurate equation of state and a reduced network of 7 isotopes from helium to nickel (Timmes et al. 2000) able to estimate the approximate nuclear energy release during the evolution. Note that as shown in Goriely et al. (2002) the use of a reduced network may underestimate the total energy release and consequently affect the thermodynamic profile of the track particles, although the isotopic yields integrated over the entire envelope remains rather unaffected. However, at the present time, it remains unconceivable to perform 3D simulations with an extended network. The initial model considered here corresponds to model A of GSBW, which is in turn a 3D map of Model 3 calculated by Woosley \& Weaver (1994). The main features of the initial model are summarized in Table 1 . We give below a short description of the hydrodynamical evolution of the WD from the formation of the first helium ignition spot until the time at which a large fraction of the nuclear binding energy content of the star has been converted into kinetic energy. More details about the evolution of the model are given in GSBW.

The explosion is triggered artificially by increasing the temperature in a tiny region located at the base of the accreted helium layer. As the material is quite degenerate a thermal runaway ensued until the temperature is high enough (currently around $T_{9} \simeq 3$, where $T_{9}$ is the temperature expressed in $10^{9} \mathrm{~K}$ ) to remove the degeneracy. At this point the heated region expands, like a piston, into the surrounding material. A strong shock wave is formed which rapidly transforms into a detonation due to the fast and energetic nuclear combustion developed at the rear edge of the shock wave. Soon a ChapmannJouguet self-sustained detonation emerged (see for instance Fig. 4 of GSBW). During its journey the substrate of the detonation slides over the carbon-helium interphase eventually reaching all the helium layer and blowing the envelope away. Approximately one second later the detonation wave converges in the antipodes of the star (with respect to the initial ignited region) where a hot spot forms. The combined rocket effects induced by the evaporation of the nuclear ashes of the incinerated helium layer and by the overcompressed region in the hot spot at the antipodes induce the carbon detonation close to (but not at) the center of the WD. This second detonation propagates all the way through the core of the star which is finally disrupted. Several observational features typical of type Ia supernovae, such as the kinetic energy or the total mass of ${ }^{56} \mathrm{Ni}$ ejected are well reproduced by this model but it shares the same drawbacks as the 1D models concerning the spectrum, i.e. in particular too much $\mathrm{Ni}$ and unburnt $\mathrm{He}$ in the outer layers.

In order to calculate the detailed nucleosynthesis from the hydrodynamical models the thermodynamical history of the system was monitored at regular times. Every $\Delta t=6 \times 10^{-3} \mathrm{~s}$, the temperature, density and other relevant physical quantities are stored for each particle from the beginning of the explosion until $t \simeq 2.55 \mathrm{~s}$ when all nuclear reactions are virtually quenched by the expansion. Even using a moderate number of particles (77712 in the present calculation) it represented a huge volume of data.

\section{Detailed nucleosynthesis}

The composition of the ejecta and core is evaluated by a post-processing nucleosynthesis calculation based on the temperature and density profiles derived from the SPH simulation. A full network including some 50000 reactions on about 4000 nuclides up to Po and lying between the proton- and neutron-drip lines is solved. All nuclear input data are taken from the Brussels nuclear reaction rate library (BRUSLIB) based on published compilations of experimental reaction rates and the determination of reaction rates within the statistical Hauser-Feshbach model (Aikawa et al. 2005). It remains however untractable at the present time to follow up all the 77712 particles of the hydrodynamical simulations with an extented network, so that a proper sampling of these particles needs to be performed. For the core and the envelope, separately, a first selection is made by considering only particles with a peak temperature $T_{9}^{\text {peak }}$ in the $1.8 \leq T_{9} \leq$ 3.8 range known to be of relevance for the production of 


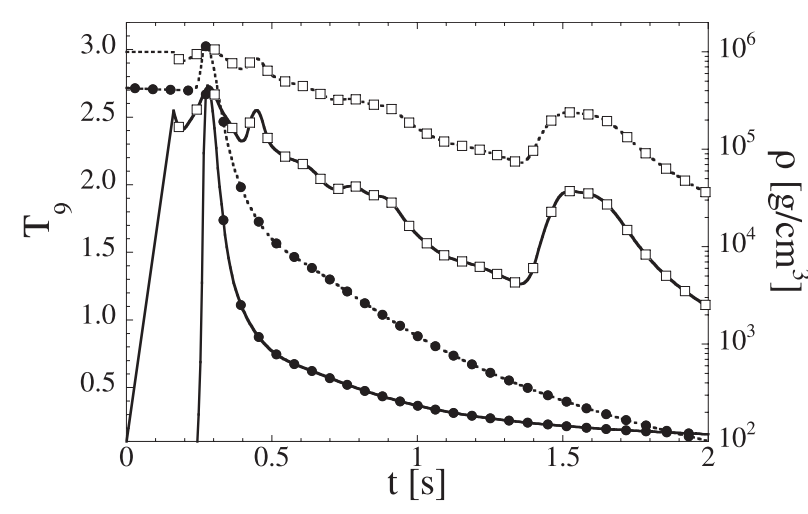

Fig. 1. Temperature (solid line) and density (dashed line) profiles for two particles with the same peak temperature $T_{9}^{\text {peak }}=2.73 \pm 0.01$. Bullets correspond to a fast expanding particle and squares to a slow expanding one.

p-process nuclei (Arnould \& Goriely 2003). This leaves us with some 21768 particles corresponding to $0.059 M_{\odot}$ in the core and $0.158 M_{\odot}$ in the envelope. These particles are then sorted in 100 bins of increasing peak temperatures. A second property of the evolutionary track of particular importance for our application concerns the expansion timescales. The longer the high temperatures are maintained, the more significant the photoerosion of the surrounding material. For this reason, for each peak-temperature bin a second sampling is carried out on the expansion time (which we arbitrarily define as being the time elapsed between the temperature peak and the one at which the temperature has dropped below $\left.T_{9}^{\text {peak }} / 1.4\right)$, so that in each peak temperature bin, the slowest and fastest expansion time are finally considered and assumed to be representative of all the other tracks within the corresponding bin. The large-network calculations are performed for these resulting 400 particles, 200 in the core and 200 in the envelope, each of the sampled bin being weighted by the total mass of the particles within the corresponding peak-temperature bin.

To illustrate the need to sample each peak-temperature bin with both a slow- and a fast-expanding particle, we show in Fig. 1 the temperature and density profiles of two particles reaching the same peak temperature of $T_{9}^{\text {peak }}=2.73 \pm 0.01$, but characterized by different expansion timescales Their resulting p-process abundance distributions are given in Fig. 2. As can be observed the long-lasting photo-irradiation tends to destroy significantly more, in particular the heavy species

Finally, based on such a sampling, i.e. twice 200 particles characteristic of the disrupted core and ejected envelope, the final composition of the ejecta is determined. Note that as in Goriely et al. (2002), the envelope is assumed to be initially He-rich (98\% in mass) and ${ }^{14} \mathrm{~N}$-rich (1\% in mass). As pointed out by Woosley \& Weaver (1994), the consideration of an initial non-zero ${ }^{14} \mathrm{~N}$ amount is critical, and is expected to result from the burning of $\mathrm{H}$ in the material accreted from the WD companion. In contrast the WD core is made of $30 \% \mathrm{C}, 69 \% \mathrm{O}$ and $1 \%{ }^{22} \mathrm{Ne}$ (Table 1). As far as the elements heavier than $\mathrm{Si}$ are concerned, both the envelope and the core are assumed to be initially of solar composition. We show in Fig. 3 the resulting abundance distributions characteristic of

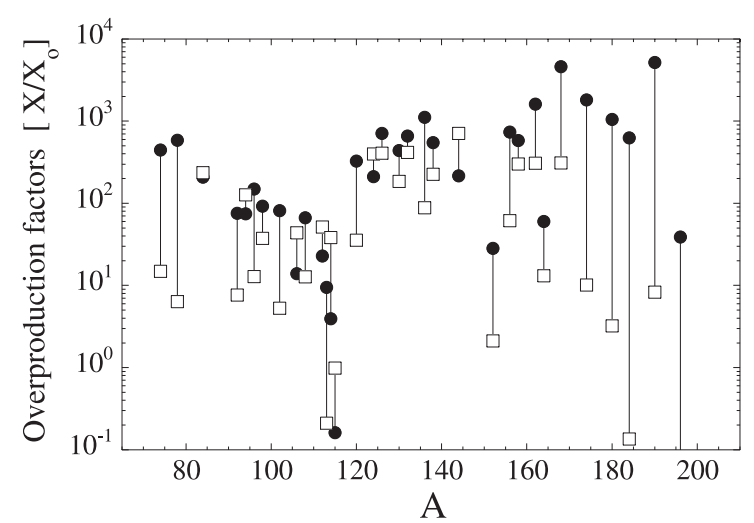

Fig. 2. Final abundance distribution of p-nuclei (with respect to the solar one) for the two particle tracks shown in Fig. 1, i.e. a fast expanding particle (bullets) and a slow expanding one (squares).

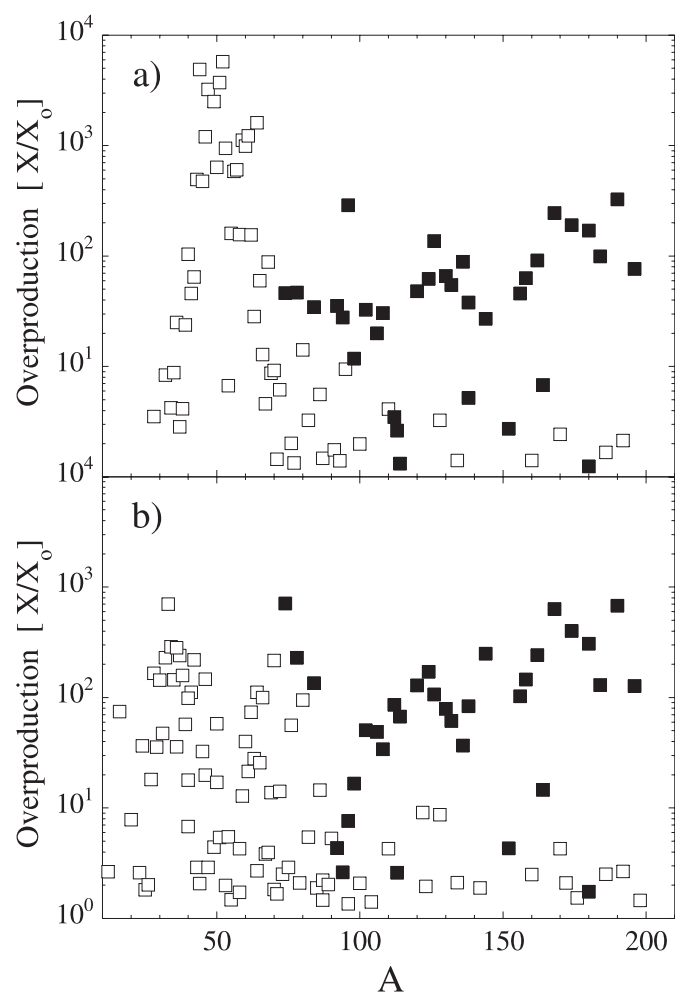

Fig. 3. a) Final composition of the ejected envelope as a function of the mass number $A$. Full symbols denote the p-nuclides. b) Same as a) for the disrupted core.

the $0.059 M_{\odot}$ core and $0.158 M_{\odot}$ envelope and in Fig. 4 the total mass-weighted distribution (note that the composition of the remaining mass has not been estimated).

As can be seen in Fig. 3, the core composition in heavy elements corresponds to a p-process distribution typical of type Ia supernova explosions (Arnould \& Goriely 2003) with a clear deficiency in the production of ${ }^{92,94} \mathrm{Mo}$ and ${ }^{96,98} \mathrm{Ru}$, but also of ${ }^{138} \mathrm{La}$ and ${ }^{180} \mathrm{Ta}$. The p-nuclei are overproduced in amount similar to the lightest species. It should however be kept in mind that the remaining $0.7 M_{\odot}$ of the WD reaches the nuclear statistical equilibrium during the explosion and is therefore expected to be composed essentially of ${ }^{56} \mathrm{Ni}$. This leads to a 


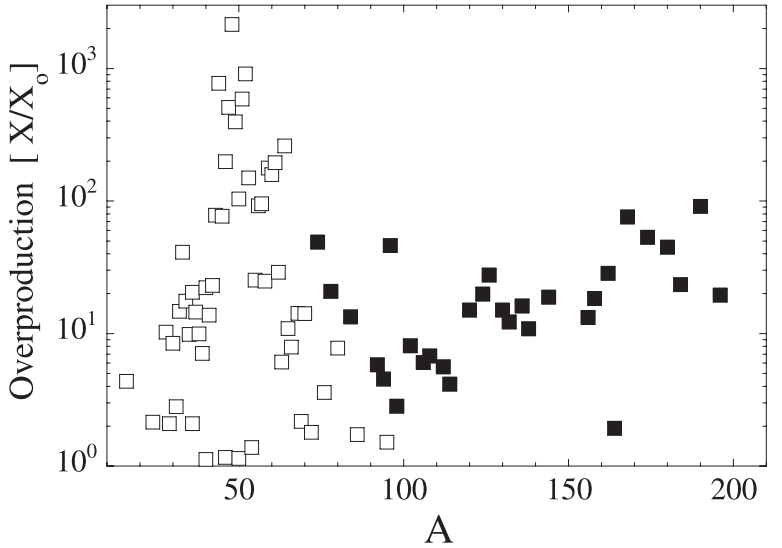

Fig. 4. Final composition of the ejected material included in the postprocessed $0.217 M_{\odot}$ envelope and core as a function of the mass number $A$. Full symbols denote the p-nuclides.

relative underabundance by a factor of about 10 of the p-nuclei shown in Fig. 3b with respect to ${ }^{56} \mathrm{Fe}$.

As far as the envelope composition is concerned, we obtain results very similar to the one found in the 1D simulation of Goriely et al. (2002, 2003). These include a large overall overproduction of p-nuclei (including ${ }^{92,94} \mathrm{Mo}$ and ${ }^{96,98} \mathrm{Ru}$ ) by a factor of about 100 . In the 3D simulation, no overproduction of the ${ }^{74} \mathrm{Se}$ or ${ }^{78} \mathrm{Kr}$ is obtained, but rather a large overabundance of ${ }^{96} \mathrm{Ru}$. These differences are essentially due to the use of the updated nuclear physics library, but also to the use of more detailed evolutionary tracks in the 3D case. The nuclear processes taking place in this scenario are described in Goriely et al. (2002) and are note repeated here. As in the 1D calculations, Fig. 3a also makes clear that the Ca-to-Fe nuclei (most particularly ${ }^{44} \mathrm{Ca},{ }^{47,49} \mathrm{Ti},{ }^{51} \mathrm{~V},{ }^{52} \mathrm{Cr}$ ) are overabundant with respect to the p-nuclei, which implies that the considered He detonation is not an efficient scenario for the production of the bulk solar-system p-nuclides. To cure this problem, as suggested in Goriely et al. (2002), we may envision a possible enhancement of the initial abundance of the s-nuclides, which are the seeds for the p-process. The plausibility of this s-process enhancement in the envelope is discussed by Goriely et al. (2002) and remains at the core of the problem to make the present site a viable one for the p-process nucleosynthesis.

\section{Conclusions}

We have carried out the first attempt to estimate the production of p-nuclei on the basis of a multi-dimensional hydrodynamical simulation. However, due to the complex nuclear flow and the extended network required for such simulations, the postprocessing nucleosynthesis calculations are restricted to a sample of well-chosen particles believed to be representative of the material enriched in p-nuclei during the explosion. It is shown that the $\mathrm{p}$-abundance distribution in the disrupted core is very similar to the one found in traditional type-Ia supernovae, with however an underabundance by a factor of about 10 with respect to the production of ${ }^{56} \mathrm{Ni}$. Similarly to the 1D simulation in Goriely et al. (2002), the envelope is found to be enriched in p-nuclei (including the puzzling Mo and Ru p-isotopes) which remain however underabundant with respect to $\mathrm{Ca}-\mathrm{Fe}$ nuclei. The underabundance problems of both the core and the envelope can be cured by considering an enhanced initial abundance of s-elements. The astrophysical plausibility of this enhancement, as well as the He-detonation of such He-accreting subChandrasekhar CO-WD and their global contribution to the galactic nuclidic content remain to be scrutinized in detail.

Acknowledgements. S.G. is FNRS research associate. This research has been partially supported by the Spanish MCyT programs AYA2002-0494-C03 and AYA2004-06290-C02-02.

\section{References}

Aikawa, M., Arnould, M., Goriely, S., Jorissen, A., \& Takahashi, K. 2005, A\&A, in press

Arnould, M., \& Goriely, S. 2003, Phys. Rep., 384, 1

García-Senz, D., Bravo, E., \& Woosley, S. E. 1999, A\&A, 349, 177

Goriely, S., José, J., Hernanz, M., Rayet, M., \& Arnould, M. 2002, A\&A, 383, L27

Goriely, S., José, J., Hernanz, M., Rayet, M., \& Arnould, M. 2003, Nucl. Phys. A, 718, 596

Iben, I. Jr., \& Tutukov, A. V. 1991, ApJ, 370, 615

Livne, E., \& Arnett, D. 1995, ApJ, 452, 62

Timmes, F., Woosley, S. E., Hartmann, D. H., \& Hoffman, R. D. 1996, ApJ, 464, 332

Timmes, F., Hoffman, R. D., \& Woosley, S. E. 2000, ApJS, 129, 377

Woosley, S. E., \& Weaver, T. A. 1994, ApJ, 433, 371

Yoon, S.-C., \& Langer, N. 2004, A\&A, 419, 645 